\title{
PRÉSTAMOS INGLESES EN MISQUITO
}

\author{
Mario Portilla
}

\begin{abstract}
The article describes the characteristics of the linguistic variety from which the English loans in Misquito, a Misumalpan language of Honduras and Nicaragua, were taken. Moreover, it describes the changes in form of the English items when they are taken in as loans.
\end{abstract}

\section{Antecedentes históricos*}

La presencia inglesa en la Mosquitia se remonta a principios del siglo XVII. En 1629 las islas de Providencia y San Andrés, las cuales se encuentran a 160 kilómetros de la costa mosquita, fueron descubiertas por el capitán inglés Sussex Cammock. Dada la importancia agrícola y estratégica de estas islas para los británicos, fue fundada allí una colonia, apoyada por fondos privados, los cuales eran admistrados por una compañía comercial denominada Compañía Providencia. Esta compañía, creada en 1630 por puritanos ingleses, tenía por objetivo ocupar cualquier lugar que no estuviera bajo el dominio español, en un área comprendida aproximadamente entre la península de Yucatán y la isla La Española, con el fin expandir el comercio británico en el Caribe occidental. En 1633 tras una expedición del mismo capitán Cammock, los ingleses crearon asentamientos permanentes en la costa del Cabo Gracias a Dios en Honduras y en Bluefields en Nicaragua ${ }^{1}$.

Cronológicamente, estas poblaciones en la Mosquitia figuran, pues, entre los asentamientos británicos más antiguos en el Caribe ${ }^{2}$.

De acuerdo con la política comercial de la Compañía Providencia, las relaciones de los ingleses con los indígenas de la Mosquitia debieron haber sido bastante estrechas y amistosas ${ }^{3}$.

En 1635 los españoles inician una serie de hostilidades en contra de la presencia inglesa en el Caribe occidental. En 1635 la isla de Providencia es atacada desde Cartagena de Indias por primera vez, pero el ataque es rechazado. Finalmente, en 1641 los españoles logran tomar la isla de Providencia, la cual estaba habitada por cuatrocientos colonos. Condenan a galeras a los 
hombres y envían a los niños y mujeres a Inglaterra. Providencia quedaría en poder de los españoles hasta 1670.

Holm (1978: 324 y ss.; 1983: 97) afirma que quizás un buen número de esclavos negros, probablemente hablantes de alguna variedad de sabir o pidgin inglés, escaparían de Providencia durante la conquista española de la isla y se habrían mezclado con indígenas misquitos de la costa nicaragüense.

La expulsión de los ingleses de sus posesiones en las costas centroamericanas sería solo temporal. En 1655, tras un fallido ataque a La Española, una expedición militar logra apoderarse de Jamaica. Esta isla se convertirá en la colonia más importante para los ingleses en la región, desde la cual partirán las principales operaciones de expansión británica en el Caribe occidental.

Las actividades inglesas en la Mosquitia estarán dedicadas sobre todo al contrabando y a la piratería.

Los piratas ingleses asolaron el Caribe hispánico, especialmente Centroamérica, entre 1665 y 1689. Durante esta época se afianza la alianza entre ingleses y misquitos. En diversas ocasiones los misquitos acompañaron a los bucaneros británicos en sus incursiones en contra de las poblaciones españolas.

Paralelamente a la piratería, hubo en toda la costa centroamericana presencia de colonos ingleses, dedicados sobre todo al corte de palo campeche. La migración inglesa a la región fue paulatinamente en aumento durante todo el siglo XVII. Especialmente en la Mosquitia proliferaron asentamientos ingleses, desde Black River en Honduras, hasta el río Maíz, al sur de Nicaragua ${ }^{4}$.

La alianza entre ingleses y misquitos fue siempre muy apreciada por ambas partes. Desde 1687 se establece incluso la costumbre de que el rey misquito fuera coronado en Jamaica. A partir de 1740 los ingleses llegan a instaurar también una especie de protectorado sobre la Mosquitia, el cual era administrado desde Jamaica.

Sin embargo, a causa de la rivalidad entre los imperios inglés y español, la disputa por la soberanía sobre la Mosquitia se extenderá durante todo el siglo XVIII. Finalmente, en 1787 los ingleses se ven obligados a evacuar la costa debido a una victoria militar española. Aunque la mayoría de los colonos británicos emigran a Belice junto con sus esclavos, una buena cantidad de hablantes de inglés o de criollo inglés permanece en la Mosquitia. El uso extendido de criollo inglés como primera lengua en la región es un hecho hasta nuestros días, dado el relativo aislamiento de la zona del resto de los países hispanohablantes a que pertenecen ${ }^{5}$.

\subsection{Propósito de la investigación}

Los hechos históricos mencionados anteriormente muestran la gran antigüedad y naturaleza muy estrecha del contacto entre anglohablantes y misquitos en la Mosquitia. Esto explica la enorme cantidad de palabras de origen inglés que existen en misquito.

El presente trabajo establece una caracterización del inglés del cual fueron tomados. Luego, ofrece una descripción de las adaptaciones o cambios lingüísticos de los rubros ingleses al ser incorporados como préstamos en misquito. 
Los materiales utilizados para el análisis son los siguientes: Una lista de 129 rubros proporcionada por Edy McNab, nativo de Puerto Lempira, en Honduras. Una lista de 53 rubros de Jairo Wood Grantwald, nativo de Brewers Lagoon, en Honduras. Estas listas fueron recogidas en diciembre de 1994 en Tegucigalpa y transcritas por el autor de este estudio. Una lista de 74 rubros, recogida por Adolfo Constenla en 1987 en San José a Maywell Padilla Héctor, nativo de Dákura, pueblo ubicado cerca de Puerto Cabezas, en Nicaragua, la cual fue transcrita por el autor de este estudio. Una lista de 511 rubros, tomada del diccionario de Marx y Heath (1961). Una lista de 150 rubros tomada del diccionario del CIDCA (1986), el cual fue confeccionado con la colaboración de informantes de Puerto Cabezas.

\section{Características del inglés usado en la Mosquitia}

Holm (1978: 24) supone la existencia de una variedad sabirizada de inglés en la Mosquitia desde principios de siglo XVII, la cual habría sido usada entre misquitos e ingleses.

La primera noticia explícita que se tiene del uso de una variedad pidginizada de inglés por parte de los misquitos es una referencia de un viajero inglés, Hans Sloane, quien visitó la región a principios del siglo XVIII. Al referirse a los indios misquitos de Cabo Gracias a Dios, en Honduras, hace el siguiente comentario: "The men generally speak broken English" (Sloane 1707: lxxvii, citado por Holm 1983: 99).

Holm (1978: 329-33) presenta algunos textos primitivos de la variedad de inglés utilizada por los misquitos. Los textos más antiguos datan de la segunda mitad del siglo XIX. Estos muestran una cierta semejanza con la variedad criolla hablada en la región; sin embargo, también exhiben rasgos diferentes a esta, atribuibles a pidginización. De estos, quizá el texto más interesante, recogido en 1847 , es uno que muestra que las formas sabirizadas eran utilizadas no solo por los misquitos, sino por los mismos ingleses.

One of them sung out, in pretty good English, 'How do? Me glad see you long time you no come' to which one of our men, who had been in the country before, and who knew the Indians, replied, 'Tokoy, plenty English come live with you, bring plenty everything, too much'. (Holm 1978: 330)

La comparación de ciertos préstamos misquitos con las formas correspondientes en los criollos ingleses del Atlántico demuestra que estos fueron tomados de la misma protovariedad que dio origen a estos criollos ${ }^{6}$.

Algunas de estas características comunes de los préstamos misquitos con las formas criollas difieren de manera importante respecto del inglés estándar.

En primer lugar, ciertos rubros están basados en la forma inglesa plural, aunque con significado singular tanto en misquito como en los criollos ingleses del Atlántico ${ }^{7}$.

MIS su(:)s ${ }^{8}$ zapato < ING shoes (cf. SRA súsu KRI sús JAM, COS, NIC, SAN, HON, BEL Ju:z idem frente a ING EST shoe).

MIS bi(:)ns frijol < ING beans (cf. KRI bínt JAM, COS, BEL binz idem frente a ING EST bean). 
MIS saks calcetín < ING socks (cf. KRI sóks COS, NIC, SAN, BEL saks idem frente a ING EST sock)

MIS tumátis tomate < ING tomatoes (cf. KRI tamátís JAM, COS, BEL, HON tomátis idem frente a ING EST tomato $\operatorname{RP}^{9}$ [ ta Ima:tau]).

Tanto en misquito como en los criollos ingleses del Atlántico existe el mismo marcador de aspecto completivo:

MIS don ${ }^{10}$ aspecto completivo < ING done (cf. KRI don, JAM, COS, NIC, SAN, HON, BEL d $\wedge$ idem ).

En misquito el rubro correspondiente a la palabra araña es de origen africano al igual que en las variedades más basilectales de los criollos ingleses del Atlántico:

MIS anánsi araña < twi ananse (cf. SRA anánsi KRI nansí JAM, COS, NIC, BEL, HON, SAN (a)nansí idem, personaje de cuentos tradicionales ).

Por otra parte, algunos de estos préstamos manifiestan el carácter arcaico o dialectal de la variedad de inglés del que fueron tomados:

MIS pu(:)s gato < ING puss (cf. SRA puspúsi11 KRI, JAM, COS, NIC, SAN, BEL pus idem frente a ING EST cat). El OED reporta esta forma a principios del siglo XVI.

MIS girg trompo < ING gig (cf. JAM, COS, NIC, SAN, HON gig idem frente a ING EST (whipping-)top). El OED reporta este significado a finales del siglo XVI. Actualmente esta forma es obsoleta o tiene un carácter dialectal.

MIS mása señorito < ING master (cf. SRA másra KRI mása JAM, COS, NIC, BEL má:sa señor (como título) frente a ING EST master RP [ 'mo:stə ]). El OED reporta el significado que aparece en misquito a finales del siglo XVI. La pronunciación que se presenta en misquito y en los criollos es arcaica o dialectal.

MIS kákaras cucaracha < ING cacaroch (cf. COS, NIC, HON kakarúotj idem frente a ING EST cockroach RP [ 'kpkrauts ] ). El OED reporta esta forma a principios del siglo XVII, la cual se convierte en obsoleta desde el siglo XIX.

MIS tsajní chino < ING chinee (cf. KRI țainí JAM, BEL, COS, NIC ţainí: idem frente a ING EST chinese). El OED reporta esta forma a mediados del siglo XVII. Actualmente, esta palabra es rara u obsoleta.

MIS pra(:)k camisa < ING frock (cf. KRI frok JAM, COS, NIC, SAN, HON, BEL frak vestido formal de mujer e ING EST shirt). El OED reporta el significado que aparece en misquito a principios del siglo XVII. Actualmente, este uso es raro u obsoleto.

MIS karibí indio garífuna (caribe) < ING Caribee (cf. ING EST Carib). El OED reporta esta forma a principios del siglo XVIII, la cual actualmente es rara u obsoleta.

MIS ku(:)l carbón < ING coal (cf. SRA krofája (< ING ARC coal of fire) KRI kol JAM, COS, NIC, HON, BEL kuol idem frente a ING EST charcoal). El OED reporta la vigencia de esta forma desde el siglo XII hasta mediados del siglo XIX.

MIS traus pantalón < ING trouse (cf. ING EST trousers). El OED reporta esta forma como singular entre finales del siglo XVII y principios del siglo XIX.

MIS tínki < ING thankee < thank-ye gracias (cf. RP ['Өæøk ju] thank you). El OED registra esta forma como dialectal a principios del siglo XIX. Este rubro se deriva presumible-

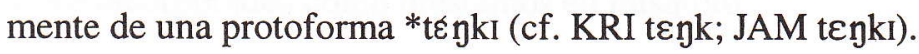


Igualmente, los préstamos reflejan algunas formas de pronunciación arcaicas, las cuales aparecen también en los criollos ingleses atlánticos.

En inglés moderno temaprano, la palatalización de / $\mathrm{k} / \mathrm{y} / \mathrm{g} /$ ante $/ æ /$ como / $\mathrm{k}^{\mathrm{j}} / \mathrm{y}$ / $\mathrm{g}^{\mathrm{j}} /$ respectivamente fue un fenómeno común, el cual sobrevivió incluso en el estándar hasta finales del siglo XVIII (Dobson 1968: § 379). Esta pronunciación sobrevive en los criollos ingleses del Atlántico. Algunos ejemplos de esta pronunciación son los siguientes:

MIS kjáptin capitán < ING captain (cf. COS kjáptın idem ).

MIS kjáput gorra < ING cap < FRA capput (cf. COS kjap idem ).

MIS kjas efectivo < ING cash (cf. COS kjas idem).

MIS kjas barril < ING cask (cf. COS kjas idem ).

MIS kjálik, gjálik ajo < ING garlic (cf. COS gjálık idem ).

MIS kjásalin gasolina < ING gasoline (cf. COS gjaslín idem ).

Igualmente, la velarización de / p / y / b / ante / $\mathrm{II} /$ como $\left[\mathrm{p}^{\mathrm{w}}\right]$ y $\left[\mathrm{b}^{\mathrm{w}}\right]$ respectivamente es otro ejemplo de una pronuciación arcaica, la cual también fue común en inglés durante el siglo XVIII (Wakelin 1988)12. La presencia de esta pronuciación en los préstamos ingleses tomados por el misquito se nota en el carácter redondeado de la vocal / u / del grupo vocálico / ui /, el cual es el reflejo del grupo [ $\left.{ }^{\mathrm{N}} \Lambda \mathrm{I}\right]^{13}$ :

MIS pújsin veneno < ING poison (cf. COS pwáızın idem ).

MIS buj boya < ING buoy (cf. COS bwar idem ).

Otro rasgo de pronunciación arcaico, propio del inglés moderno temprano, es la pérdida de la aproximante / $\mathrm{w} /$ delante de la vocal / $\mathrm{u} /$ (Wakelin 1988) ${ }^{14}$.

MIS piroman mujer de mala vida < ING spare woman (cf. NIC peir úman prostituta; SRA úma; KRI úman; JAM, COS, NIC, BEL, HON, SAN úman mujer ).

MIS man-ud especie de árbol (literalmente madera macho ) < ING man + wood (?) RP [wud] (cf. SRA údu; KRI JAM, COS, NIC, BEL, SAN ud madera ).

\section{Adaptaciones fonológicas de los préstamos ingleses al misquito}

\subsection{El sistema fonológico del misquito}

El sistema vocálico del misquito tanto hondureño como nicaragüense exhibe dos rasgos distintivos estables: [ \pm bajo], [ \pm posterior] (cf. cuadro 1).

\section{Cuadro 1}

Sistema de oposiciones vocálicas estables en misquito

[-posterior]

[-bajo]

[+bajo] [+posterior]

$\mathbf{u}$ 
Ejemplos de apariciones de estas vocales en misquito son los siguientes:
/ i/ li agua
/ u / dus árbol
/ a / ba en

Además, el sistema vocálico presenta tres rasgos distintivos inestables: [ \pm alto], [ \pm nasal], [ \pm largo] (cf. cuadro 2).

\section{Cuadro 2}

Sistema de oposiciones vocálicas inestables en misquito

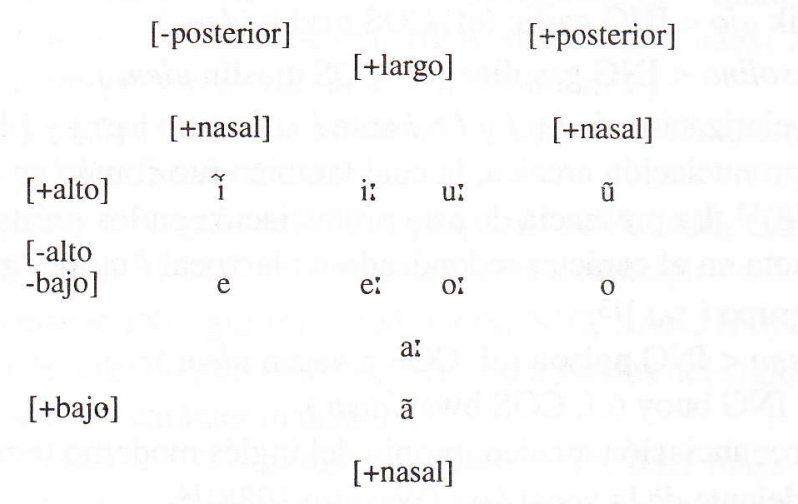

Ejemplos de apariciones de estas vocales en misquito son los siguientes ${ }^{15}$ :

/ e / ten diez

/ o goloro

/ i: / ki candado

/ u: / buits bota

/ ai/ sais harpón

/ e:/ se:r silla

/ o:/ go:ts cabra

/ il inajallorar

/ ũ / sũ roble

/ ã / ãwaja flotar

Las vocales cortas medias / e / y / o / se presentan solamente en palabras de origen extranjero, las cuales presumiblemente son préstamos más recientes, aún no adaptados totalmente a la fonología del misquito. Por esta razón, aparecen algunos dobletes del mismo étimo con diferentes pronunciaciones, por ejemplo, gol gul $\sim$ kul oro $(<\mathrm{ING}$ gold), god $\sim$ gad $\sim$ kat Dios (< ING God), kwerko kwirku cerdo (< ESP puerco).

Por otra parte, existen discrepancias entre los distintos informantes en cuanto a la cantidad vocálica asignada a ciertos rubros. Así, por ejemplo, ambos informantes hondureños distinguen por cantidad el siguiente par mínimo de esta forma: sus zapato: su:s sarna. Los diccionarios del CIDCA y Marx y Heath (1961) asignan la cantidad de manera inversa: su:s zapato: sus sarna. El infor- 
mante nicaragüense, por su parte, ofrece ambas palabras con vocal larga: sus zapato, sarna.

En otro caso, el informante nicaragüense, al igual que el diccionario del CIDCA, distingue otro par mínimo por cantidad así: wis decir (imp.): wis (kati) setiembre. Sin embargo, el diccionario de Marx y Heath (1961) asigna la cantidad al par mínimo de manera inversa: wiis decir (imp.) : wis kati setiembre.

También existen discrepancias entre los informantes en cuanto a la asignación de la nasalidad a ciertos rubros. Algunos ejemplos de estas discrepancias son los siguientes:

\begin{tabular}{|c|c|c|c|c|}
\hline Jairo Wood (Hon.) & Maywell Padilla (Nic.) & CIDCA & \multicolumn{2}{|c|}{ Marx y Heath (1961) } \\
\hline kúa & kũa ${ }^{16}$ & kua & khua & piojo \\
\hline $\mathrm{ma}$ & mã & ma: & $\mathrm{ma}$ & semilla \\
\hline mála & mãla & mala & mala & afilado \\
\hline pihní & pîhni & pihni & pihni & blanco \\
\hline smih & smin & smih & smih & izquierda \\
\hline tã:ski & tãski & ta:ski & taski & sucio \\
\hline kĩãma & kjamã & kjama & kiama & oreja \\
\hline ínska & inska & inska & inska & pez \\
\hline ãwaja & - & awaja & awaja & flotar \\
\hline ãhhkiká & ãjhkika & aihkika & aihkika & derecha \\
\hline- & sũ & - & usupum & roble \\
\hline
\end{tabular}

El sistema consonántico del misquito presenta los siguientes rasgos distintivos: [ \pm con-

Cuadro 3

Sistema de oposiciones consonánticas en misquito

$$
\begin{array}{lll}
\text { [tanterior }] & {[\text {-anterior }]} \\
\text { [-coronal }] & {[\text { +coronal }]} & {[\text {-coronal }]}
\end{array}
$$

[+consonante]:

$\begin{array}{rrrr}{[\text { [-sonoro }]} & \mathrm{p} & \mathrm{t} & \mathrm{k} \\ {\left[\begin{array}{c}\text { [-continuo] } \\ {[\text { sonoro }]}\end{array}\right.} & \mathrm{b} & \mathrm{d} & \end{array}$

[+obstruyente]

[+continuo]

[+nasal]

m

S

[-obstruyente]

[+lateral]

[-nasal]

[-lateral] r

[+consonate]: 
sonante], [ \pm bajo], $[ \pm$ posterior] (cf. cuadro 3).

Ejemplos de apariciones de estas consonantes en misquito son los siguientes:

$\begin{array}{ll}\text { / p / } & \text { pása viento } \\ \text { / t } & \text { tára grande } \\ \text { / k / } & \text { káhula frío } \\ \text { / b / } & \text { ba en } \\ \text { / d / } & \text { dábaja chupar } \\ \text { / s / } & \text { sáwra malo } \\ \text { / m / } & \text { málas desafilado } \\ \text { / n / } & \text { nákra ojo } \\ \text { / g/ } & \text { jạ yo } \\ \text { / l/ } & \text { lájkra hermana } \\ \text { / } / & \text { rája nuevo } \\ \text { / w / } & \text { wálpa piedra } \\ \text { / j/ } & \text { jạyo } \\ \text { / h / } & \text { máhbra huevo }\end{array}$

Por último, en cuanto a los suprasegmentales, el misquito presenta un acento de intensidad no distintivo, el cual aparece en posición inicial de palabra. Además, esta lengua exhibe una oposición distintiva entre un tono alto y un tono bajo. A continuación se ofrecen algunos ejemplos de rubros polisílabos con una distribución de patrones tonales distinta.

$\begin{array}{ll}\text { tásba tierra } & \text { (alto-bajo) } \\ \text { búhni seco } & \text { (alto-bajo) } \\ \text { pawtá fuego } & \text { (bajo-alto) } \\ \text { matsíb cinco } & \text { (bajo-alto) } \\ \text { káhula viento } & \text { (alto-bajo-bajo) } \\ \text { kasbriká nube } & \text { (bajo-bajo-alto) } \\ \text { asmalá garra } & \text { (bajo-bajo-alto) } \\ \text { papíki padre } & \text { (bajo-alto-bajo) }\end{array}$

\subsection{El sistema fonológico del protocriollo inglés del Atlántico}

El protocriollo inglés tal como ha sido reconstruido por Portilla (1994: capítulo 3) presenta un sistema de 6 vocales cortas, 6 vocales largas y 3 diptongos. El sistema vocálico presenta las siguientes oposiciones en términos de rasgos distintivos: [ \pm anterior], [ \pm alto], [ \pm bajo] y [ \pm largo] (cf. cuadro 4). 


\section{Cuadro 4 \\ Sistema vocálico del protocriollo inglés del Atlántico \\ [+anterior] [-anterior] \\ [-largo $] \quad[+$ largo $] \quad[+$ largo $] \quad[-1$ largo $]$}

$\begin{array}{lllll}\text { [+alto] } & \text { I } & \text { i: } & \text { u: } & u \\ \begin{array}{c}\text {-alto } \\ \text {-bajo] }\end{array} & \varepsilon & \text { e: } & \text { o: } & \Lambda \\ \text { [+bajo] } & \text { a } & \text { ai: } & \text { o: } & \text { D }\end{array}$

Diptongos: $\quad$ al $\Lambda \mathrm{I} \quad \Lambda \mathrm{U}$

Además, el protocriollo inglés del Atlántico presenta un sistema de 8 consonantes oclusivas, 5 fricativas, 3 nasales, 2 líquidas y 3 paravocales.

Este sistema exhibe las siguientes oposiciones de rasgos distintivos: [ \pm consonante], [ \pm obstruyente], $[ \pm$ sonoro], $[ \pm$ continuo $],[ \pm$ nasal $]$ y $[ \pm$ lateral $]$ (cf. cuadro 5 ).

[+consonante]

\section{Cuadro 5}

Sistema consonántico del protocriollo inglés del Atlántico

$\begin{array}{llll} & {[\text { +anterior }]} & & {[\text {-anterior }]} \\ \text { [+ consonante } & {[\text {-coronal }]} & {[\text { +coronal }]} & {[\text {-coronal }]}\end{array}$

[-sonoro] $\quad \mathrm{p} \quad \mathrm{t} \quad \mathrm{t} \quad \mathrm{k}$

[-continuo]

$\begin{array}{llllll}\text { [+sonoro }] & \text { b } & \text { d } & \widehat{d}_{3} & \mathrm{~g}\end{array}$

[+obstruyente]

$\begin{array}{ccccc}{[\text {-continuo] }} & \text { [-sonoro }] & \mathrm{f} & \mathrm{s} & \mathrm{s} \\ {[\text { +sonoro }]} & \mathrm{v} & \mathrm{z}\end{array}$

$[+$ nasal $] \quad m \quad n$

[-obstruyente]

[+lateral]
$[$-nasal $]$
$[$-lateral $]$


Por último, en cuanto a los suprasegmentales, Portilla (1994: 133 y ss.) postula que el protocriollo inglés del Atlántico era o bien una lengua de acento dinámico o bien una lengua de acento tonal, que presentaba una oposición entre un tono alto y un tono bajo. En todo caso, este autor reconstruye solamente un tono alto o acento por palabra polisilábica.

\subsection{Reflejo de los fonemas del protocriollo inglés del Atlántico en misquito}

\subsubsection{Las vocales cortas con tono alto o en posición acentuada} $*_{\mathbf{I}}>\mathbf{i}^{17}$

il ( $<$ hill) cerro, siks ( $<$ six) seis, dínar (< dinner) almuerzo, comida, $\sin (<\sin )$ pecado, mil (< mill) molino, kik (< kick) patear, tsrim $(<$ shrimp) camarón.

$*_{\boldsymbol{u}}>\mathbf{u}$

búlit (< bullet) bala, buk (< book) libro, pus (< puss) gato, gutnajt (< good night) buenas noches, súgar (< sugar) azúcar, súkaplun (< sugar + plun = comida) confite .

$*_{\varepsilon}>\mathbf{i}$

bil ( $<$ bell) campana, brid (< bread) pan, bil $(<$ belt) cinturón, íbans $(<$ Evans) apellido, sins $(<\mathrm{sens})$ sentido, razón, twínti $(<\mathrm{twenty})$ veinte, ris $(<\mathrm{rest})$ descanso.

$>\mathrm{e}$

bel ( $<$ bell) campana, ten $(<$ ten) diez, sében $(<$ seven) siete, hel $(<$ hell) infierno, mériki ( $<$ American) estadounidense, pénsil ( $<$ pencil) lápiz, bens $(<$ bench) banca .

$* \wedge>\mathbf{u}$

bútar (< butter) mantequilla, bútarflaj (< butterfly) mariposa, drum (< drum) tamborcillo, nurs (< nurse) enfermera, súbil (< shovel) pala, rum (< rum) aguardiente, purs (< purse) cartera.

$>\mathbf{a}$

wark (< work) trabajo, wark tákaja (work + tákaja = acontecer) trabajar, kap (< cup) taza, trábil (< trouble) problema, kas (< curse) mala palabra, pas (< first) primero, hántin tákaja ( $<$ hunting + tákaja= acontecer) cazar.

La realización redondeada de / u / como reflejo de la protovocal $*_{\Lambda}$ parece estar favorecida por el contacto con una consonante bilabial ${ }^{18}$.

$* \mathbf{a}>\mathbf{a}$

an (< and) y, ámar (< hammer) martillo, kjáptin (< captain) capitán, kjásalin (< gasoline) gasolina, kjáput (< cap(put)) gorra, tag (< tag) colilla, tiquete, blánkit $(<$ blanket) cobija . *o > a

áhsa (< ox) hacha, ándris (< orange) naranja, baks (< box) caja, sap (< shop) tienda, bátil (< bottle) botella, dálar (< dollar) dólar, lábsta (< lobster) langosta.

\subsubsection{Vocales cortas en posición inacentuada o con tono bajo}

Para el protocriollo inglés del Atlántico han sido reconstruidas las siguientes vocales cortas en posición inacentuada: ${ }^{*} \mathrm{I},{ }^{*} \varepsilon, *_{U}, *_{\Lambda}$ y $*_{\partial}$ (Portilla 1994: 105-7). Desgraciadamente, en algunos casos, los datos disponibles ofrecen pocos ejemplos de palabras con los reflejos de ta- 
les vocales.

$*_{\text {I }}>$ i

búlit (< bullet) bala, áspital (< hospital) hospital, ínglis (< English) inglés, mánin (< morning) buenos días, blánkit (< blanket) cobija, mériki (< American) estadounidense, kápi $(<$ coffee) café.

$* \varepsilon>e$

sében (< seven) siete. Cf. SAR sében, JAM seben.

$>a$

(h)ándat (< hundred) cien. Cf. JAM, COS, NIC, SAN, BEL Ándred idem.

$* \Lambda>\mathbf{a}$

wínda (< window) ventana. Cf. KRI wíndo, JAM, COS, BEL wínd idem.

*ə> $\mathbf{a}$

ámar (< hammer) martillo, kwábas (< guava) guayaba, árba (< harbor) puerto, pístal (< pistol) pistola, sígarit (< cigarret) cigarrillo, marít (< married + tákaja= acontecer) casarse, lagún (< lagoon) lago, laguna.

\subsubsection{Vocales largas}

En los informantes hondureños, no existe una plena consistencia en la atribución de la cantidad vocálica. Además, en el idiolecto de uno de los informantes, Jairo Wood, la aparición de vocales largas estaba generalmente correlacionada con la presencia de nasalidad: [tãski] sucio, [kî:] candado.

En el material del informante nicaragüense, es posible distinguir diferencias de cantidad en los rubros recogidos por Constenla. En cuanto a los rubros que son préstamos de origen inglés, existe una correspondencia de cantidad con respecto del término original en aproximandamente un $80 \%$ de los casos (cf. Apéndice 3).

Los diccionarios del CIDCA y de Marx y Heath (1961) exhiben una situación semejante a la descrita para el informante nicaragüense.

Esta situación puede explicarse de dos formas diferentes. Por una parte, podría postularse que en misquito, principalmente en la variedad hondureña, la inconsistencia en la distinción de cantidad se debe a un cambio en proceso, cuya tendencia es la eliminación de la cantitad vocálica como rasgo distintivo. Por otra parte, podría pensarse que la preservación de la distinción de cantidad se deba a la mayor influencia de adstrato que tiene el criollo inglés de Nicaragua sobre el misquito nicaragüense.

Dadas las diferencias entre el misquito hondureño y el nicaragüense en cuanto a la cantidad mencionadas anteriormente, se presentan a continuación los reflejos de las protoformas en las dos variedades por separado.

\section{$*_{\mathrm{i}:}>$ i $(\mathbf{H O N})$}

bins ( $<$ beens) frijol, bip ( $<$ beef) vaca, ki (< key) candado, pri $(<$ free) libre, tri $(<$ three) tres, sípar (< cheaper) barato, ti $(<$ tea) té.

$>$ I: 
$*_{\mathrm{i}}>\mathrm{i}$ (NIC)

kî: (< key) cayo, islote.

$>\mathbf{i}$ ki: $(<$ key) cayo, llave, kwin (< queen) reina.

$*$ u: $>$ u (HON)

pil ( $<$ field) campo, bins ( $<$ bean) frijol.

bruslagún ( $<$ Brewers Lagoon) topónimo, kul (< school) escuela, marún ( $<$ maroon) cimarrón, lagún (< lagoon) lago, mjúsik (< music) música, sus (< shoes) zapato, blu (<blue) azul.

$*_{\mathrm{u}}>>$ u: (NIC)

bu:ts (< boots) bota, sun (< soon) temprano, juis (< use) uso, su:s (< shoes) zapato.

$>\mathbf{u}$

$*_{\mathrm{e}}>\mathrm{i}(\mathrm{HON})$

bir (< beer) cerveza ${ }^{19}$, krísi (< crazy) loco, plit (< plate) plato, sir (< chair) silla, it $(<$ eight) ocho, trínsar ( $<$ stranger) extraño, mistík ( $<$ mistake) error.

$>\mathrm{e}$

$*_{\mathrm{e}}$ > ii (NIC)

méri (< Mary) nombre propio 20.

i.t (< eight) ocho.

$>$ i

kik (<cake) queque.

$*_{\text {o: }}>$ u $($ HON $)$

dur ( $<$ door) puerta, rup (< rope) mecate, snu ( $<$ snow) nieve, túri (< story) cuento, gul $(<$ gold $)$ oro, núkaja $(<$ know + kaja $=$ ser $)$ saber .

$>0$

gol (< gold) oro, por (< four) cuatro.

*o: $>$ u: (NIC)

guits (< goats) cabra, hurr (< whore) puta, nu: (< know) saber, purp (< Pope) Papa, ru:p $>\mathbf{u}$ (< rope) mecate, buttar (boat + tar) brea de bote.

kum (< comb) peine, sup (< soap) jabón.

$*_{\mathrm{a}}>\mathbf{a}(\mathbf{H O N})$

$*_{a}:>$ a: (NIC)

dans (< dance) baile, glas (< glass) vaso, luganglas (< looking glass) espejo.

kjait (< card) carta, la:t (< lard) manteca, ma:s (< mast) mástil, da:ns pulaja $(<$ dance + pulaja $=$ jugar $)$ bailar, ha:p $(<$ half $)$ mitad .

$*_{\text {o: }}>$ a $($ HON $)$

áras ( $<$ horse) caballo, bal ( $<$ ball) bola, gad ( $<$ God) Dios, árba ( $<$ harbor) puerto, la $(<$ law) ley, mánin (< morning) buenos días, sámil (< sawmill) aserradero.

$>$ a:

sa: (< saw) serrucho.

$*_{\text {J: }}>$ a: (NIC) 
ba:s (< boss) jefe, sa: (< saw) serrucho, war (<war) guerra.

$>\mathbf{a}$

bal ( $<$ ball) bola

\subsubsection{Los diptongos}

*ar $>$ aj

ajs (< ice) hielo, najn (< nine) nueve, pajn (< fine) bueno, lajt (< light) luz, rajs (< rice) arroz, tsajní (< chinee) chino, rajpl (< rifle) rifle.

$*_{\mathbf{A I}}>\mathbf{a j}$

ajl (< oil) aceite.

$>$ uy $/ *$ pw-

*bw

pújsin (< poison) veneno, buj (< buoy) boya 21 .

$*_{\Lambda} \boldsymbol{>}>$ aw

báwman (< bowman) piloto de cayuco, traws (< trouse) pantalón, áwar (< hour) hora, pláwar (< flour) harina, páwtara (< pouder) polvo, táwan (< town) ciudad, dawn (< town) pueblo.

\subsubsection{Las consonantes}

$* \mathrm{p}>\mathrm{p}$

pus (< puss) gato, prámas (< promise) promesa, kap (< cup) taza, rup (< rope) mecate, map (< map) mapa, kjáptin (< captain) capitán, sípar (< cheaper) barato.

$* \mathbf{b}>\mathbf{b}$

bal (< ball) bola, búlit (< bullet) bala, blánkit (< blanket) cobija, íspun (< spoon) cuchara, lábsta (< lobster) langosta, kínkrab (< king + crab) tipo de pez, kárib (< carib) garífuna. $* \mathbf{t}>\mathbf{t}$

táwan ( $<$ town) ciudad, tu ( $<$ two) dos, dáktar ( $<$ doctor) doctor, tri $(<$ three) tres, kat $(<$ cot) catre, sígarit (< cigarret) cigarillo, gu:ts (< goats) cabra 22 .

$* \mathbf{d}>\mathbf{d}$

dáktar (< doctor) doctor, dínar (< dinner) comida, dras (< drawers) calzoncillo, drum (< drum) tamborcillo, gad ( $<$ God) Dios, wínda ( $<$ window) ventana.

\section{$>\mathbf{t}$ (especialmente en posición final de palabra)}

la:t (< lard) manteca, ándat (< hundred) cien, marít (< married) cónyuge, páwtara (< pouder) pólvora.

$* \mathbf{k}>\mathbf{k}$

ká:pi (< coffee) café, ki: (< key) cayo, islote, klim munaja (< clean + munaja = hacer) limpiar, saks (< socks) calcetín, kik (< kick) patear, kwin (< queen) reina, blánkit (< blanket) cobija 23 .

$>\mathbf{h}$

áhsa (< ax) hacha.

$* \mathrm{~g}>\mathrm{g}$

lagún (< lagoon) lago, laguna, súgar (< sugar) azúcar, glas (< glass) vaso, gad (< God) 
Dios, gul (< gold) oro, gítar (< guitar) guitarra, sígarit (< cigarret) cigarrillo .

$>\mathbf{k}$

lakún (< lagoon) lago, laguna, súkaplun $(<$ sugar + plun = comida) confite, kwábas $(<$ guavas) guayaba.

$* \mathrm{f}] \mathrm{s}$

sis (<chest) cofre, $\sin (<$ chain) cadena, sípar (< cheaper) barato, sir (< chair) silla, kákaras ( $<$ kakaroach) cucaracha.

$>$ ts

tsajní ( $<$ chinee) chino, mats ( $<$ match) fósforo, kítsin $(<$ kitchen $)$ cocina. $* d_{3}>\mathrm{s}$

tsins ( $<$ change) cambio, trínsar (< stranger) extraño, ándris (< orange) naranja, pásis (< passage) pasaje.

$* \mathbf{f}>\mathbf{p}$

pas (< first) primero, pil (< field) campo, kápi $(<$ coffee) café, prak ( $<$ frock) camisa, bip ( $<$ beef) vaca, pajb $(<$ five) cinco, por $(<$ four $)$ cuatro 24 .

$* \mathrm{v}>\mathrm{b}$

kwábas (< guavas) guayaba, plantinriba (< planting + river) topónimo, sében $(<$ seven) siete, pajb ( $<$ five) cinco, íbans ( $<$ Evans) nombre propio.

$*_{\mathrm{s}}>\mathrm{s}$

sa: (< saw) serrucho, sében ( $<$ seven) siete, mistík ( $<$ mistake) equivocado, místar $(<$ mister) señor, kjásalin (< gasoline) gasolina, ris $(<$ rest $)$ descanso.

$>\emptyset$

kul (< school) escuela, túri (< story) cuento, trínsar (< stranger) extraño.

La ausencia de la consonante / s / en posición inicial ante una consonante oclusiva sorda, refleja probablemente la situación original del protocriollo inglés del Atlántico (cf. v.gr. COS tuorí (< story) cuento, kápjan (< scorpion) escorpión ).

$* \mathrm{z}>\mathrm{s}$ zapato.

mjusik ( $<$ music) música, traws ( $<$ trouse) pantalón, bins ( $<$ beens) frijol, su:s $(<$ shoes)

$* \int>\mathrm{s}$

súgar ( $<$ sugar) azúcar, súkaplun (sugar + plun = comida) confite, mísin $(<$ machine $)$ máquina, su:s ( $<$ shoes) zapato, kjas (< cash) efectivo, ínglis ( $<$ English) inglés.

$* \mathbf{h}>\mathbf{h}$

hándat ( $<$ hundred) cien, ha:p ( $<$ half) mitad, hil $(<$ hill) cerro, hur ( $<$ whore) puta, hel $(<$ hell) infierno.

$\emptyset$

ándat ( $<$ hundred) cien, il ( $<$ hill) cerro, ámar $(<$ hammer) martillo, áras $(<$ horse) caballo, árba (< harbor) puerto, árikin (< hurricane) huracán, áspital (< hospital) hospital.

Aunque es posible reconstruir una consonante *h para el protocriollo inglés del Atlántico, se debe señalar que la mayoría de las variedades criollas exhiben la misma alternancia / h$\varnothing /$ que muestra el misquito.

$* \mathrm{~m}>\mathrm{m}$

milk (< milk) leche, místar (< mister) señor, krísmis (< christmas) navidad, mériki $(<$ 
American) estadounidense, sámil (< sawmill) aserradero, drum (< drum) tamborcillo, dajm $(<$ dime) 20 centavos.

*n $>$ n

najt (< night) noche, nurs (< nurse) enfermera, dans (< dance) baile, síknis ( $<$ sickness) enfermedad, dínar (< dinner) comida, sében (< seven) siete, wan (< one) uno 25 .

$*_{\eta}>\eta$

kin (< king) rey, kinkrab (< king + crab) tipo de pez, mánin (< morning) buenos días, ran (< wrong) error, ínglis (< English) inglés.

$* 1>$ I

la (< law) ley, luganglas (< looking glass) espejo, plit $(<$ plate) plato, dálar $(<$ dollar $)$ dólar, ólikira (< holly + kira el que hace) santo, sámil (< sawmill) aserradero, il (< hill) cerro. $*_{\mathbf{r}}>\mathbf{r}$

rum (< rum) aguardiente, rajs (< rice) arroz, nurs (< nurse) enfermera, marún (< maroon) cimarrón, krísi (< crazy) loco, áwar (< hour) hora, dáktar (< doctor) doctor.

$\varnothing$

mánin (< morning) buenos días, lábsta (< lobster) langosta, plantinriba $(<$ planting + river) topónimo, súkaplun $(<$ sugar + plun = comida $)$ confite .

En posición posvocálica, la presencia o la ausencia de la consonante / -r / refleja una situación de variación que existía probablemente en el protocriollo inglés del Atlántico (cf. Portilla 1994: 130-1). En esta posición, la conservación de la consonante / -r / alcanza 23 casos (18 en posición final de palabra y 5 ante consonate) frente a 9 de elisión de esta (4 en posición final de palabra y 5 ante consonante).

$*_{\mathrm{w}}>\mathrm{w}$

wan (< one) uno, wark (<work) trabajo, wári (< war) guerra, wínda (< window) ventana, áwar (< hour) hora, kwin (< queen) reina, twínti $(<$ twenty) veinte.

$* \mathbf{j}>\mathbf{j}$

ju:s (< use) uso, mjúsik (< music) música, kja:t (< card) carta de naipe, kjásalin (< gasoline PCIA *kjásəlın) gasolina.

\subsubsection{Los suprasegmentales}

En misquito, el acento o tono alto reconstruido para el protocriollo inglés del Atlántico tiene como reflejo un tono alto, mientras que la ausencia de acento o tono bajo corresponde a un tono bajo. A continuación se presenta una lista de palabras que muestran algunos patrones de distribución tonal correspondientes a la asignación del acento o tono en la protolengua.

*lóbstə(r) > lábsta langosta (alto - bajo)

*húndred > ándat cien (alto - bajo)

*kít In > kítsin cocina (alto - bajo)

*lagún > lakún lago, laguna (bajo - alto)

*maré:d > marít casarse (bajo - alto)

*misté:k > mistík equivocado (bajo - alto)

\subsubsection{Algunos casos especiales de derivación}


Algunos rubros presentan algunas características especiales que deben ser analizadas de manera particular.

En primer lugar, existe un grupo de préstamos que presenta una vocal no etimológica epentética, paragógica o protética:

*ho:rs > áras (horse) caballo

*tıun $>$ táwan (town) ciudad

* oks > áhsa (ax) hacha

* púudər > páwtara (pouder) pólvora

*spúm > *íspən > íspan (spoon) cuchara, cf. SRA spun, KRI spun, COS spunn.

En misquito, el fenómeno de epéntesis se presenta todavía a nivel fónetico en ciertos casos, por ejemplo, máhbra ['máhabra] huevo, búhni ['búhuni] seco, wáhja ['wáhaja] hoja, pihní ['pihiní] blanco.

Una posible explicación del fenómeno anterior es suponer que estos préstamos fueron adaptados en una época temprana del contacto anglo-misquito.

\section{Conclusiones}

Uno de los aspectos más interesantes que pone en evidencia el presente estudio es que los préstamos ingleses que aparecen en misquito son tomados de la misma protovariedad que dio origen a los criollos ingleses del Atlántico. En estos criollos ingleses así como en el misquito, la protovariedad de inglés refleja un carácter dialectal y arcaico tanto en pronunciación como en el significado de ciertos rubros léxicos.

Por otro lado, se puede señalar que la abundancia de léxico de origen inglés en misquito ha producido una situación de inestabilidad en el sistema fonológico sobre todo en cuanto a las vocales. De esta forma, el vocabulario de origen patrimonial presenta solamente la oposición entre tres vocales. El léxico de origen inglés presenta un número mayor de contrastes entre vocales. Estas oposiciones no tienen, sin embargo, un carácter estable, sino que dan lugar a una situación de variación de pronunciaciones, inclusive en un mismo hablante.

En la mayoría de los casos, los reflejos de los protofonemas que aparecen en los préstamos ingleses tomados en misquito presentan una gran regularidad. Sin embargo, unos pocos protofonemas exhiben una variación en los reflejos que producen. Esto ocurre sobre todo con las vocales. Esto puede deberse o bien a la situación de inestabilidad fonológica descrita anteriormente o bien a que los préstamos fueron tomados en distintas épocas.

Notas

* Deseo expresar mi agradecimiento a Adolfo Constenla por haberme suministrado la grabación del material que aparece en el apéndice 3 y a Annette Calvo por su colaboración en la descripción fonética de parte del material utilizado. 
1. El nombre de este puerto es la anglización del apellido holandés de un miembro de la Compañía Providencia, Blauvelt ("A forgotten Puritan Colony”. Blackwood's Edinburg Magazine. CLSV, No MIII: 878, Mayo 1899, citado por Floyd 1967(1990): 25).

2. El primer asentamiento en el Caribe fue establecido en Bermuda en 1609. Luego, en 1624 y 1627 son fundadas las colonias de St. Kitts y Barbados respectivamente. Barbados se convertirá en el punto de partida más importante para la expansión inglesa en el Caribe. Desde aquí son fundadas las colonias de Surinam en 1651 y de Jamaica en 1655. Cf. Holm 1986.

3. Cf. una carta de 1632 citada por Holm 1978: 25.

4. Floyd 1967(1990): 60-1.

5. Holm (1983: 98) calcula que la población de hablantes de criollo inglés como primera lengua en la Mosquitia es de 40.000 personas.

6. Hancock (1969) fue el primero en señalar el origen común de las variedades criollas inglesas que existen a ambos lados del Atlántico. Según Holm (1988: xvi-xvii) el número de estas variedades asciende a 27, distribuidas en Norteamérica, Centroamérica, el Caribe, Suramérica y la costa occidental de África.

7. Las variedades criollas inglesas tomadas en cuenta para la comparación son el sranan (SRA) de Surinam (Echteld 1961), el krio (KRI) de Sierra Leona (Fyle y Jones 1980) y los criollos ingleses de Jamaica (JAM) (Hancock 1969, Holm 1978, Wells 1973), Costa Rica (COS), Nicaragua (NIC), San Andrés y Providencia (SAN), Honduras (Islas de la Bahía) (HON), y Belice (BEL) (Holm 1978 y material de trabajo de campo recogido por el autor).

8. Para la transcripción fonemática se utilazan los símbolos del el Alfabeto Fonético Internacional. El símbolo ['] representa un tono alto en todas las variedades excepto en sranan, en donde representa un acento. El tono alto no se marca ni en las palabras monosilábicas de ninguna variedad ni en algunas polisilábicas del misquito, en las cuales no se ha podido determinar con exactitud la distribución de los tonos.

9. Según Wells 1990.

10. Marx y Heath 1961: 24 .

11. En esta lengua, esta palabra proviene presumiblemente de la reduplicación de la forma *pus. La vocal / i / es epentética y y su introducción obedece a una regla general de esta lengua, por medio de la cual se evita la presencia de consonantes finales (cf. Portilla 1994: 77), v.gr. PCIA *dains > SRA dánsi bailar, *fait > féti pelear, *nck > néki cuello, etc.

12. Wakelin (1988: 640) ofrece algunos ejemplos de la escritura de la época que reflejan la aparición de este fenómeno: bway muchacho y pwonting apuntar.

13. En otros contextos, el protodiptongo / * II / tiene como reflejo el diptongo / ai /; por ejemplo, ING oil > MIS ail aceite (cf. COS arl idem).

14. Algunos ejemplos de la escritura de la época que reflejan esta pronunciación son los siguientes (Wakelin 1988: 640): oman mujer y ode madera.

15. El autor ha recogido de los informantes hondureños dos ejemplos aislados de vocales largas nasales: tãiski sucio y kî̉ cayo. En los datos del informante nicaragüense ha aparecido solo un caso: wãat querer.

16. Este rubro fue suministrado al autor por Adolfo Constenla (comunicación personal).

17. Los ejemplos misquistos son tomados del material transcrito por el autor a los tres informantes, el cual aparece en los apéndices 1,2 y 3 . Estos ejemplos se presentan acompañados de la forma ortográfica inglesa estándar y la correspondiente glosa en castellano.

18. Algo semejante ocurre con los reflejos del protodiptongo * $\mathrm{\Lambda}$ (cf. el apartado 2.3.4. más adelante). 
19. Esta forma proviene en el protocriollo inglés del Atlántico de *berr, cf. NIC, HON, BEL be`r, JAM, COS bier.

20. Esta forma proviene en el protocriollo inglés del Atlántico de *mérrı, cf. NIC, HON, BEL mé:rI, JAM, COS mierí.

21. Estas formas provienen en el protocriollo inglés del Atlántico de *pwúisin y *bwai cf. COS pwásin, bwái.

22. En los datos aparece un caso de $* t>$ d, dawn $(<$ town) pueblo, el cual puede explicarse como una ultracorrección.

23. Un caso de *k>g, luganglas (< looking glass) espejo puede ser explicado como una ultracorrección.

24. Uno de los informantes ofreció también las formas fajb y for.

25. En un caso la protoconsonante ${ }^{*}$ n tiene como reflejo la nasalidad: *wa:nt $>$ wãit querer.

\section{Abreviaturas}

BEL Criollo inglés de Belice

CIDCA Centro de Investigación y Documentación de la Costa Atlántica (de Nicaragua)

COS Criollo inglés de Costa Rica

ESP Español

HON Criollo inglés de las Islas de la Bahía, Honduras

Hon. Honduras

imp. Modo imperativo

ING ARC Inglés arcaico

ING EST Inglés estándar

ING Inglés

JAM Criollo inglés de Jamaica

KRI Krio (Sierra Leona)

MIS Misquito

NIC Criollo inglés de Nicaragua

Nic. Nicaragua

PCIA Protocriollo inglés del Atlántico

RP Received Pronunciation (Pronunciación británica estándar)

SAN Criollo inglés de San Andrés y Providencia

SRA Sranan (Surinam)

\section{Bibliografía}

CIDCA Diccionario elemental miskito-español / español-miskito. Managua: MIDINRA.

Echteld, J. 1961. The English words in Sranan. Groningen: Wolters.

Fisiak, J. (ed.). 1988. Historical dialectology. Berlín: Mouton de Gruyter.

Floyd, Troy. 1967(1990). La mosquitia: un conflicto de imperios. San Pedro Sula: Centro Editorial. 
Fyle, C. \& E. Jones. 1980. A Krio-English dictionary. Nueva York: Oxford University Press.

Görlach, Manfred \& John Holm (eds.). 1986. Focus on the Caribbean. Amsterdam: Benjamins.

Hancock, Ian. 1969. "A provisional comparison of the English-based Atlantic Creoles". African Language Review. 8: 7-72.

Holm, John. 1978. "The Creole English of Nicaragua's Miskito Coast". Tesis doctoral: University College, Nueva York.

1983. “Nicaragua’s Miskito Coast Creole English”. En: Holm (ed.), 1983: 95-130.

1986."The spread of English in the Caribbean area". En: Görlach \& Holm (eds.), 1986: 1-22.

1988. Pidgins and Creoles. Cambridge: Cambridge University Press.

Holm, John (ed.). 1983. Central American English. Heidelberg: Gross.

Marx, W. \& G. Heath. 1961. Diccionario miskito-español / español-miskito. Bethlehem: The Moravian Church in America.

Portilla, Mario. 1994. Reconstrucción fonológica y del sistema de TMA del protocriollo inglés del Atlántico. Tesis doctoral: Universidad de Bielefeld.

Sloane, Hans. 1707. A voyage to the Islands Madera, Barbados, Nieves, S. Christophers and Jamaica with a Natural History... Londres.

Wakelin, M. 1988. “The phonology of South-Western English 1500-1700”. En: Fisiak (ed.), 1988: 609-44.

Wells, John. 1973. Jamaican Pronunciation in London. Oxford: Blackwell.

1990. Longman pronuntiation dictionary. Harlow: Longman.

\section{APÉNDICE 1}

\section{Préstamos ingleses en misquito de Honduras}

Total: 129

Informante: Edy McNab

Lugar de nacimiento: Puerto Cabezas, Honduras

Recopilador: Mario Portilla 
Fecha de recopilación: Diciembre de 1994

Lugar: Islas de la Bahía y Tegucigalpa

áhsa $(<\mathrm{ax})$ hacha.

ajl (< oil) aceite

ámar (< hammer) martillo

anánsi ( $<$ anansi $<$ twi ananse) araña

ándris (< orange) naranja

áras (< horse) caballo

árba (< harbor) puerto

árikin $(<$ hurricane) huracán

áspital (< hospital) hospital

áwar (< hour) hora

bájsikil ( $<$ bicycle) bicicleta

bal $(<$ ball $)$ bola

bátil ( $<$ bottle) botella

báwman (< bowman) piloto de bote

bil $(<$ belt) faja

bill $(<$ bell) campana

bins ( $<$ beens) frijoles

bip ( $<$ beef) vaca, ganado

bir $(<$ beer $)$ cerveza

bísnup ( $<$ bishop) obispo

blánkit (< blanket) cobija

brid $(<$ bread) pan

brus lagún ( $<$ Brewers Lagoon) Brewers Lagoon (topónimo)

búlit $(<$ bullet) bala

but $(<$ boat $)$ bote

bútar $(<$ butter) mantequilla

butarflaj ( $<$ butterfly) mariposa

tsajni $(<$ chinese) chino

tsins ( $<$ change) cambiar, cambio

tsrim $(<$ shrimp) camarón

dajm ( $<$ dime) 20 centavos

dáktar ( $<$ doctor) doctor

dálar (< dollar) dólar

dans pulaja $(<$ dance + pulaja jugar, mover $)$ bailar

dans ( $<$ dance) baile

daun $(<$ town) pueblo

dínar (< dinner) almuerzo, comida

dras ( $<$ drawers) calzoncillo

dur, durunta $(<$ door) puerta

gad (<god) Dios

gítar (< guitar) guitarra 


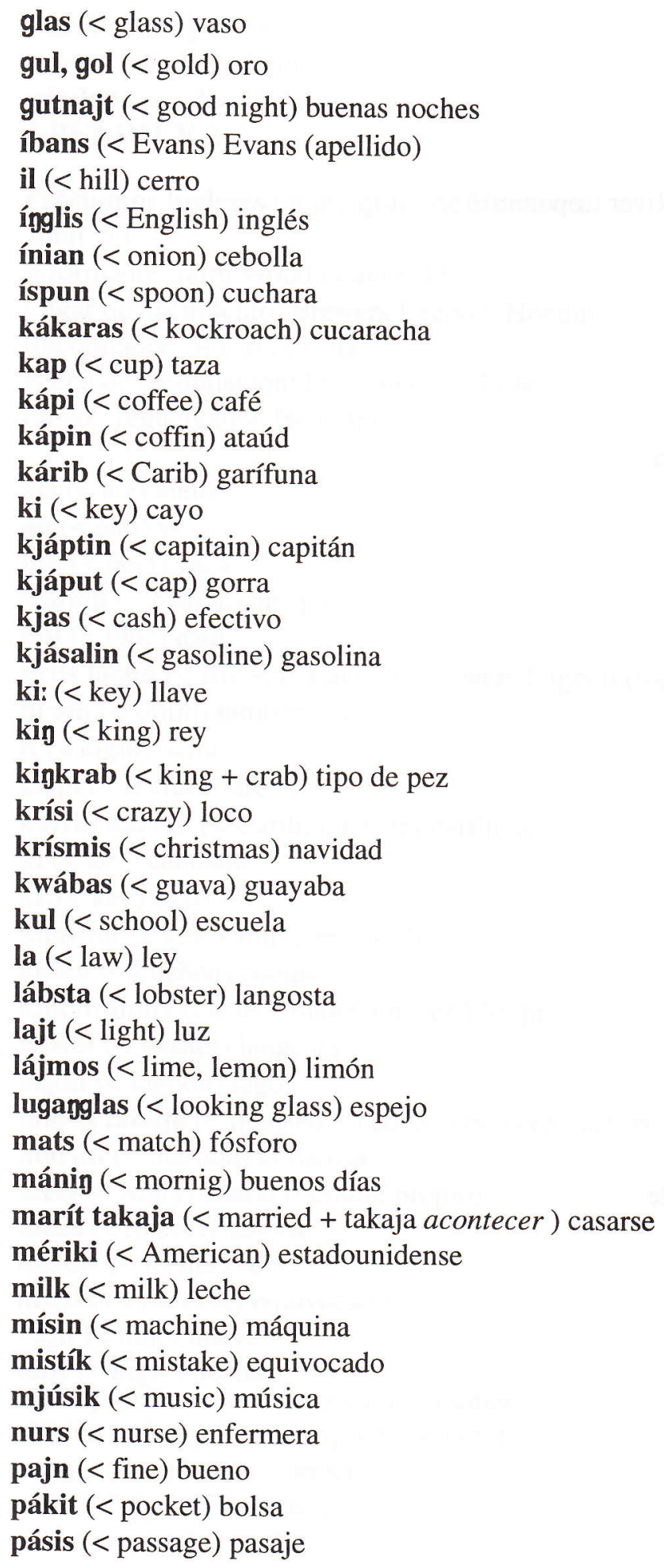




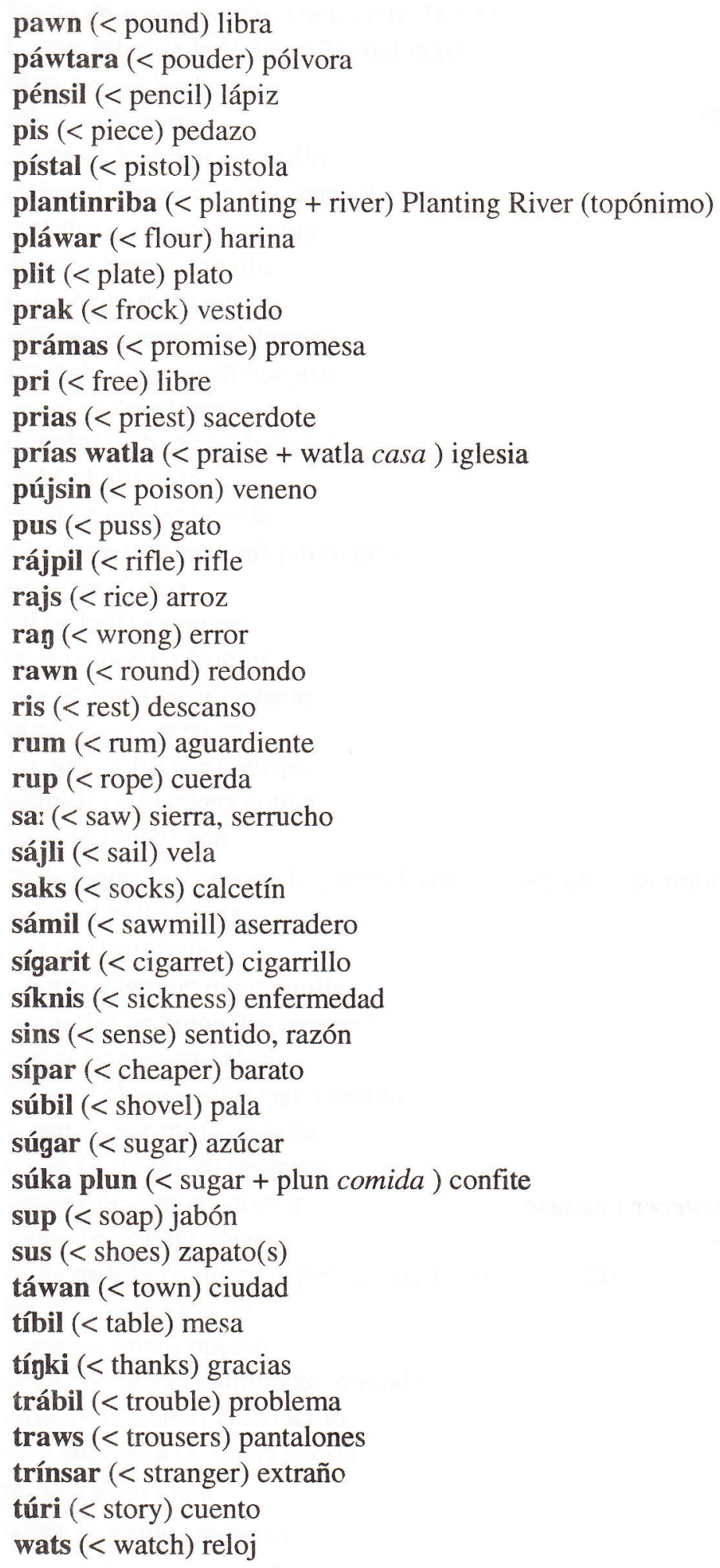


wári $(<$ war) guerra

wark ( $<$ work) trabajo

wínda ( $<$ window) ventana

\section{APÉNDICE 2}

Préstamos ingleses en misquito de Honduras

Total: 53

Informante: Jairo Wood Grantwald

Lugar de nacimiento: Brewers Lagoon, Honduras

Recopilador: Mario Portilla

Fecha de recopilación: Diciembre de 1994

Lugar: Tegucigalpa, Honduras

ajs (< ice) hielo

an $(<$ and $) y$

bip ( $<$ beef) vaca

bísnup (< bishop) obispo

blu ( $<$ blue) azul

brus lagún ( $<$ Brewers Lagoon) Brewers Lagoon (topónimo)

drum $(<$ drum) tamborcillo

it $(<$ eight) ocho

ká:pi (< coffee) café

karib, karibi. (< Carib, Caribee) garífuna

ki $(<$ key) candado

ki: (< key) cayo

kimihta $(<$ key + mihta mano ) llave

kítsin (< kitchen) cocina

klin munaja (clean + munaja hacer) limpiar

lábsta (< lobster) langosta

lagún (< lagoon) lago

marid takaja (< married + takaja acontecer ) casarse

marun ( $<$ maroon) cimarrón

meri (< Mary) María (nombre propio)

mísis ( $<$ misses) señora

místar $(<$ mister $)$ señor

mistík (< mistake) equivocado

najn ( $<$ nine) nueve

najt ( $<$ night) noche

nu kaja (< know + kaja ser, estar ) saber

olikira (< holly + kira el que hace) santo

pajn (< fine) bueno, correcto

pajp fajp (< five) cinco

pístal (< pistol) pistola

por $\sim$ for ( $<$ four) cuatro 


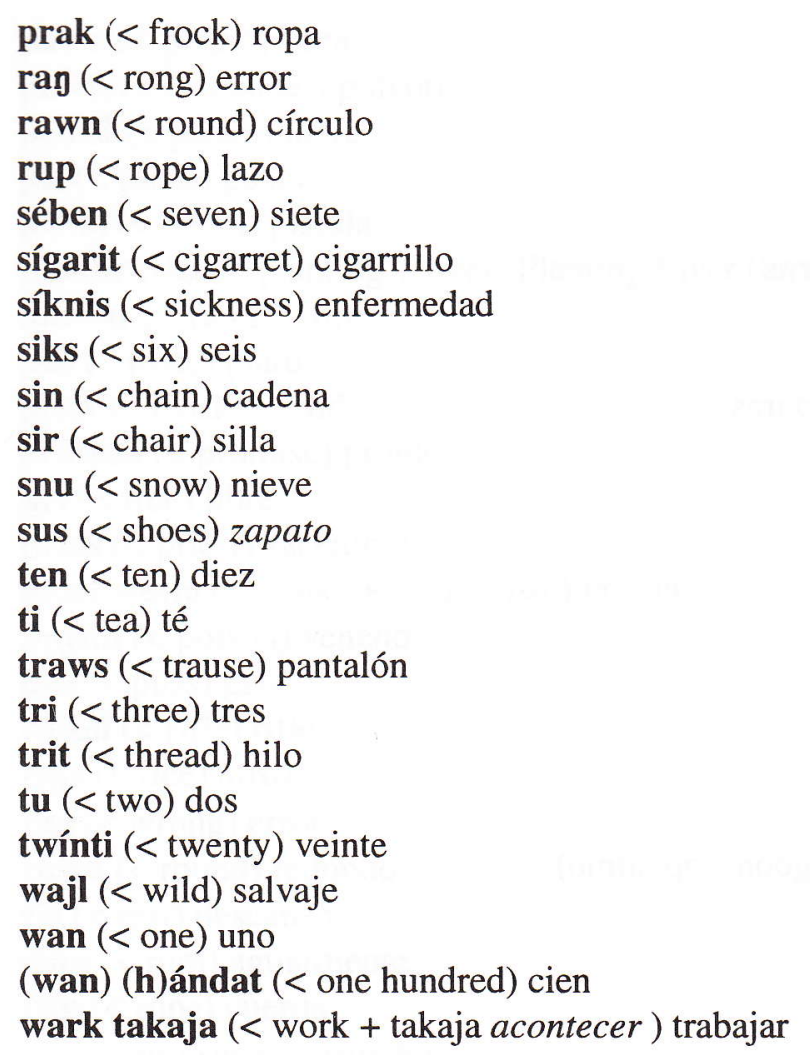

\section{APÉNDICE 3}

\section{Préstamos ingleses en misquito de Nicaragua}

\section{Total: 74}

Informante: Maywell Padilla Héctor

Lugar de nacimiento: Dákura, Nicaragua

Recopilador: Adolfo Constenla

Fecha de recopilación: 1985

Lugar: San José, Costa Rica

$$
\begin{aligned}
& \text { ajs }(<\text { ice) hielo } \\
& \text { ajs }(<\text { ice) nieve } \\
& \text { bas }(<\text { boss) jefe } \\
& \text { baks }(<\text { box) caja } \\
& \text { bal }(<\text { ball) bola }
\end{aligned}
$$


bel $(<$ bell) campana

bents $(<$ bench) banca

bil ( $<$ bell) campana

bins ( $<$ beens) frijoles

bui (< buoy) boya

buk (< book) libro

bum (< bomb) bomba

buts $(<$ boot $)$ bota

tsek ( $<$ check) cheque

dans pulaja (< dance + pulaja jugar) bailar

fab (<Fab) Fab (marca de jabón)

fal (< Fal) Fal (marca de rifle)

gig (< gig) trompo

guts (< goat) cabra

hantin takaja (hunting + takaja acontecer) cazar

hap ( $<$ half) mitad

hel $(<$ hell) infierno

hil ( $<$ hill) montaña

hur ( $<$ whore) puta

i.t (< eight) ocho

kap (< cup) taza

kas (< curse) mala palabra

kat $(<\cot )$ catre

kjat (< card) carta de naipe

ki: (< key) llave, cayo, islote

kik (< kick) patear

kik (< cake) queque

klin dawkaja (clean + dawkaja hacer) limpiar

kum $(<$ comb) peine

kwin (< queen) reina

la:t $(<$ lard) aceite

lakun ( $<$ lagoon) lago, laguna

map (< map) mapa

marit (<married) cónyuge

mas (< mast) mástil

mil $(<$ mil) molino

mok (<mug) pichel

mum (<mumps) paperas

nãin (< nine) nueve

nu ( $<$ know) saber

pãi (< fine) bueno

pas (< first) primero

pil (< field) campo 


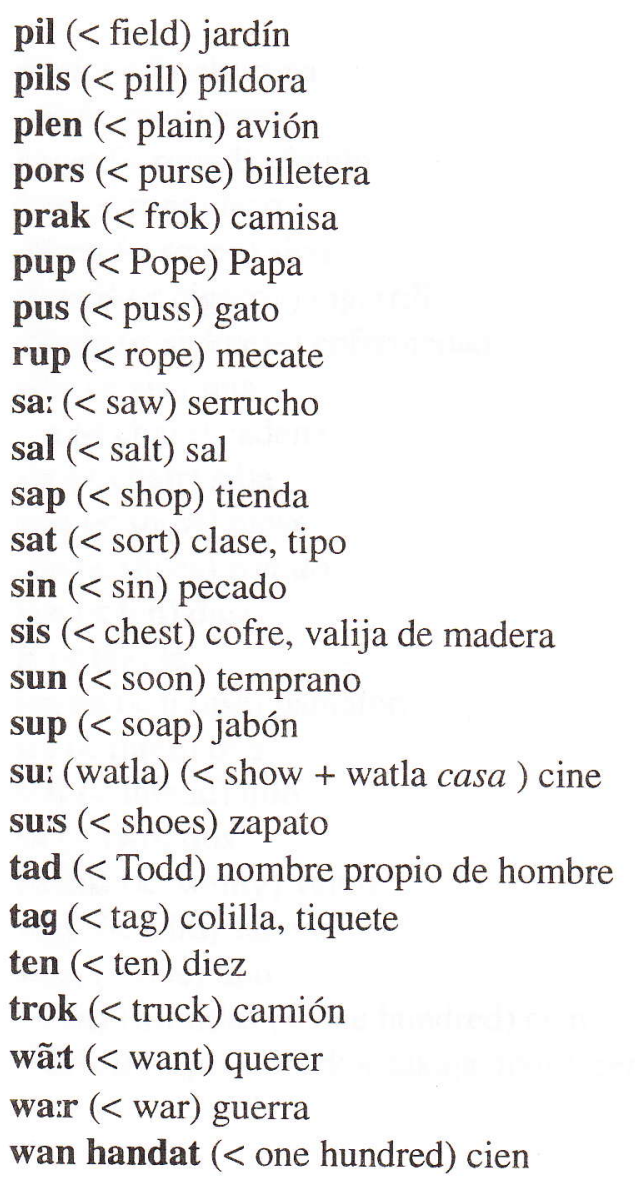

